\title{
On a possible connection between Chandler wobble and dark matter
}

\author{
Oyanarte Portilho* \\ Instituto de Física, Universidade de Brasília, \\ 70919-970 Brasília-DF, Brazil
}

(Received on 17 july, 2007)

\begin{abstract}
Chandler wobble excitation and damping, one of the open problems in geophysics, is treated as a consequence in part of the interaction between Earth and a hypothetical oblate ellipsoid made of dark matter. The physical and geometrical parameters of such an ellipsoid and the interacting torque strength is calculated in such a way to reproduce the Chandler wobble component of the polar motion in several epochs, available in the literature. It is also examined the consequences upon the geomagnetic field dynamo and generation of heat in the Earth outer core.
\end{abstract}

Keywords: Chandler wobble; Polar motion; Dark matter.

\section{INTRODUCTION}

A 305-day Earth free precession was predicted by Euler in the 18th century and was sought since then by astronomers in the form of small latitude variations. F. Küstner in 1888 and S. C. Chandler in 1891 detected such a motion but with a period of approximately 435 days. Later it was shown by Love [1] and Larmor [2] that such a disagreement between theory and observation is in part consequence of elastic deformation of the Earth which was not considered by the Euler rigid body model (see Smith and Dahlen [3] for further corrections explanation). The so called Chandler wobble (CW) is actually one of the major components of the Earth polar motion, together with the annual wobble (AW) which has period of nearly 365 days. The composition of the two wobbles results in a motion with period of around 6.2 years due to the beat phenomenon. The ever increasing measurement precision, for which techniques like very long baseline interferometry (VLBI), global positioning system (GPS) and lunar laser ranging (LLR) are employed today, has lead to the conclusion that both wobbles have varying amplitudes. For AW, this may be attributed to seasonal displacement of atmospheric and water masses. On the other hand, excitation and damping of the Chandler wobble has become a puzzle to investigators. Several mechanisms have been proposed for its excitation like snow, hidrological, atmospheric and oceanic mass displacements, and large earthquakes, with defenders and opponents arguing for and against repeatedly and, in view of the large energy involved, the problem is still an open question. In more recent papers, Gross [4] attributes the excitation to ocean bottom pressure variations and changes in ocean currents due to winds while Seitz et al. [5] focus on combined effects due to atmospheric and oceanic causes.

Since it is believed that dark matter (DM) and dark energy composes around $96 \%$ of the mass of the Universe we investigate in this paper the possibility of DM to contribute, at least in part, for the excitation and damping of Chandler wobble. The effect of DM upon regular matter has been considered mainly on galactic-scale although more recently some authors have studied smaller scale effects. For instance, Froggatt and Nielsen [6]) have investigated the possibility of star-scale effects due to small DM balls; Frère et al. [7] studied the pres-

*Electronic address: portilho@fis.unb.br ence of a DM halo centered in the Sun and its influence upon the motion of planets; numerical simulations due to Diemand et al. [8] show the presence of DM clumps surviving near the solar circle; Adler [9] suggests that internal heat production in Neptune and hot-Jupiter exoplanets is due to accretion of planet-bound, not self-annihilating DM. Although the nature of the particles that form DM is not known precisely (see for instance Khalil and Muñoz [10]) we assume that, as usual, those particles do not interact with regular matter through electromagnetic force but only gravitationally. Furthermore, we speculate that they can interact with each other in such a way to set up an oblate (due to rotation) ellipsoid that might be present inside Earth, occupying the same space without violating any physical law. It can be shown that such an ellipsoid, made of self-interacting DM (otherwise such a structure would not be possible), would be attached to Earth through a springlike restoring force. In this line, excitation and damping of $\mathrm{CW}$ could be, at least partially, consequence of energy exchange between the two bodies, associated to the restoring torque between them. The model does not consider the other known sources for the process nor the Earth internal structure, what are of course over simplifications. However this becomes possible a simple starting point for the calculations, to be sophisticated in a later step. Höpfner $[11,12]$ has managed to isolate the $\mathrm{CW}$ and $\mathrm{AW}$ components from available polar motion data. Our goal is to reproduce Höpfner's results for Chandler wobble in various epochs by adjusting the unknown physical and geometrical parameters of the dark matter ellipsoid. For this purpose, we describe both body motion by solving numerically the set of differential equations that emerge from Euler equations and demonstrate that $\mathrm{CW}$ can be reproduced in such manner. This is explained in the next section while results and discussion are presented in section III.

\section{THE MODEL}

We start by calculating the torque that arises when the polar principal axes 3 and $3^{*}$ of two oblate ellipsoids with isotropic densities interacting through the gravitational force are tilted by an angle $\alpha$ (see Fig. 1). The gravitational potential at a point outside the internal ellipsoid (see $e . g$. Stacey [13]) is given by

$$
V(r, \theta) \approx-\frac{G M^{*}}{r}+\frac{G}{r^{3}}\left(C^{*}-A^{*}\right) P_{2}(\cos \theta)
$$




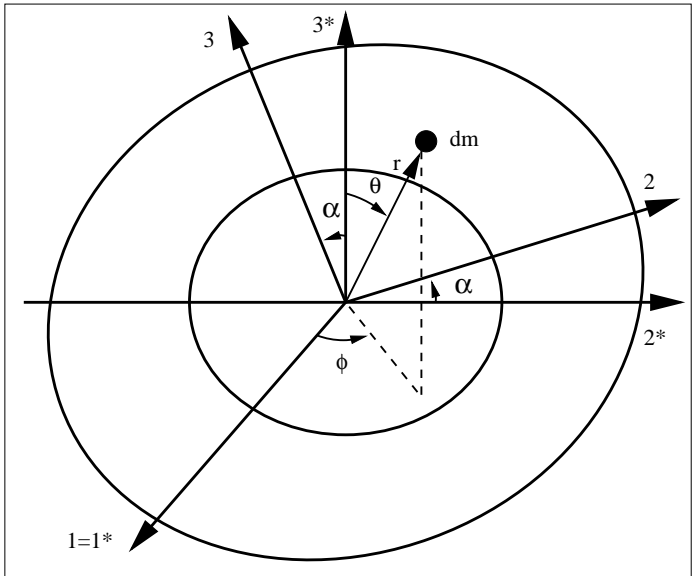

FIG. 1: Two ellipsoids tilted by an angle $\alpha$ around the common equatorial principal axes 1 and $1^{*}$. The spherical coordinates $(r, \theta, \phi)$ that give the position of an infinitesimal mass $d m$ with respect to the $1^{*} 2^{*} 3^{*}$ reference frame are also shown.

neglecting higher order terms. Here $M^{*}, A^{*}$ and $C^{*}$ are the mass, equatorial and polar momenta of inertia of the internal ellipsoid, respectively, and $P_{2}(\cos \theta)=\left(3 \cos ^{2} \theta-1\right) / 2$ is the Legendre polynomial of second order. The torque over an infinitesimal mass $d m$ located at that point is then

$$
d \tau=-d m \frac{\partial V}{\partial \theta}=\frac{3 G\left(C^{*}-A^{*}\right)}{2 r^{3}} \sin (2 \theta) d m .
$$

and is directed orthogonal to the plane formed by the polar principal axis $3^{*}$ and the position $r$ of $d m$ (see Fig. 1). Con- sidering the Earth tilted by an angle $\alpha$ around the 1-axis (Fig. 1) with respect to the internal ellipsoid it suffers therefore a torque given by the integral

$$
\tau=\int \sin \phi d \tau
$$

directed towards the identical axes 1 and $1^{*}$, as projected by the presence of $\sin \phi$ in this integral. The integration should in principle be performed over the entire Earth volume. However considering the Earth density as isotropic, as it is done in the Preliminary Reference Earth Model - PREM (Dziewonski and Anderson [14]), it can be shown that the spherical volume internal to the Earth with radius the same as the polar radius does not contribute to the integral. Therefore the integration over $r$ ranges from $R_{p}$, the polar radius, to the radius of the geoid at a given direction. On the other hand, it is well known (see, for instance, Stacey [13]) that the equation of the geoid is written in the first order approximation in terms of the co-latitude $\theta^{\prime}$ as

$$
r_{g} \approx a\left(1-f \cos ^{2} \theta^{\prime}\right)
$$

where $a$ is the equatorial radius and $f=1-R_{p} / a$ is the flattening. Let us write above equation for the case where the geoid is tilted by an angle $\alpha$ around the 1-axis (Fig. 1), which yields

$$
r_{g} \approx a\left[1-f(\cos \alpha \cos \theta-\sin \alpha \sin \theta \sin \phi)^{2}\right]
$$

where $\phi$, together with co-latitude $\theta$, is part of the spherical coordinates in the $1^{*} 2^{*} 3^{*}$ reference frame. Therefore the torque given by equation (3) becomes

$$
\begin{aligned}
\tau= & \frac{3}{2} \rho G\left(C^{*}-A^{*}\right) \int_{0}^{\pi} d \theta \sin (2 \theta) \sin \theta \\
& \times \int_{0}^{2 \pi} d \phi \sin \phi \ln \left\{\frac{a}{R_{p}}\left[1-f(\cos \alpha \cos \theta-\sin \alpha \sin \theta \sin \phi)^{2}\right]\right\}
\end{aligned}
$$

where $\rho$ is the average Earth mass density in that region, taken as $2600 \mathrm{~kg} / \mathrm{m}^{3}$ [14]. Considering that $f=1 / 298.257<<1$, we can use the approximation $\ln (1+x) \approx x$, obtaining

$$
\tau=c_{1} \sin (2 \alpha) \text {, }
$$

with $c_{1}=\frac{4 \pi}{5} \rho G\left(C^{*}-A^{*}\right) f$. Notice that this is a restoring torque when $\alpha$ is around $90^{\circ}$, which gives the stable angular position if no initial angular momenta were involved.

We describe the Earth-ellipsoid motion by the Euler equations

$$
\begin{aligned}
& A \dot{\omega}_{1}+(C-A) \omega_{2} \omega_{3}=\tau_{1} \\
& A \dot{\omega}_{2}-(C-A) \omega_{1} \omega_{3}=\tau_{2} \\
& C \dot{\omega}_{3}=\tau_{3}=0 \Rightarrow \omega_{3}=\text { constant } \\
& A^{*} \dot{\omega}_{1^{*}}^{*}+\left(C^{*}-A^{*}\right) \omega_{2^{*}}^{*} \omega_{3^{*}}^{*}=\tau_{1}^{*} \\
& A^{*} \dot{\omega}_{2^{*}}^{*}-\left(C^{*}-A^{*}\right) \omega_{1^{*}}^{*} \omega_{3^{*}}^{*}=\tau_{2}^{*} \\
& C^{*} \dot{\omega}_{3^{*}}^{*}=\tau_{3}^{*}=0 \Rightarrow \omega_{3^{*}}^{*}=\mathrm{constant}
\end{aligned}
$$

where $A$ and $C$ are the equatorial and polar momentum of inertia of Earth, which is supposed to be axially symmetric $(B=A), \omega_{1}, \omega_{2}, \omega_{3}$ are the components of the Earth angular velocity $\boldsymbol{\omega}$ in the 123 reference frame, which is attached to Earth such that the 3-axis coincides with the polar principal axis, $i$. e., the symmetry axis of the oblate ellipsoid, 1and 2 -axis are equatorial principal axes and $\tau_{1}, \tau_{2}$ are components of the torque suffered by Earth. Similar internal ellipsoid quantities are denoted by an asterisk. Notice that $\tau_{3}$ and $\tau_{3}^{*}$ vanish, what implies that $\omega_{3}$ and $\omega_{3^{*}}^{*}$ are constant. Notice also that we are not considering external torques, mainly exerted by the Sun and the Moon, which produce the precession of the equinoxes and is of no interest here. On the other hand, when $\tau_{1}=\tau_{2}=\tau_{1}^{*}=\tau_{2}^{*}=0$ equations (8)-(13) lead to the well known Eulerian free precession motion.

We write the angular velocities in terms of the Euler angles $\theta, \phi, \psi$ and their derivatives in time, defined with respect to a 


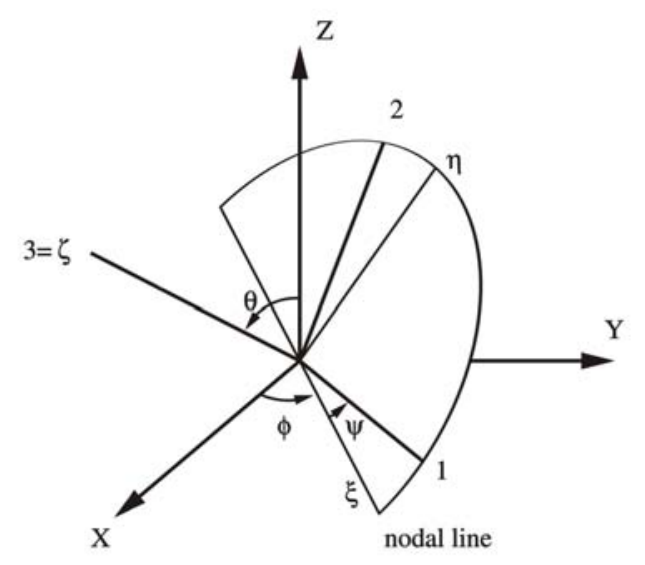

FIG. 2: Definition of the Euler angles $\theta, \phi, \psi$. fixed reference frame with axes X, Y, and Z (see Fig. 2). This frame is constructed such that the total angular momentum of the Earth-ellipsoid system, which is a constant of the motion, coincides with the $\mathrm{Z}$-axis. The relations are the following

$$
\begin{aligned}
& \omega_{1}=\dot{\theta} \cos \psi+\dot{\phi} \sin \theta \sin \psi \\
& \omega_{2}=-\dot{\theta} \sin \psi+\dot{\phi} \sin \theta \cos \psi \\
& \omega_{3}=\dot{\phi} \cos \theta+\dot{\psi}=\mathrm{constant} \Rightarrow \dot{\psi}=\omega_{3}-\dot{\phi} \cos \theta(16)
\end{aligned}
$$

Similar relations for $\omega_{1^{*}}^{*}, \omega_{2^{*}}^{*}, \omega_{3^{*}}^{*}$ can be written in terms of the Euler angles $\theta^{*}, \phi^{*}, \psi^{*}$, referred also with respect to the $\mathrm{XYZ}$ fixed system, and their derivatives in time. Notice that equation (16) serves for the purpose of eliminating $\psi$, while the same elimination can be done for $\dot{\psi}^{*}$. The components of the torque $\tau$ over the Earth can also be expressed in terms of the Euler angles

$$
\begin{aligned}
\tau_{1}= & 2 c_{1} \cos \alpha\left[-\sin \theta \cos \psi \cos \theta^{*}+(\cos \theta \cos \phi \cos \psi-\sin \phi \sin \psi) \sin \theta^{*} \cos \phi^{*}\right. \\
& \left.+(\cos \theta \sin \phi \cos \psi+\cos \phi \sin \psi) \sin \theta^{*} \sin \phi^{*}\right] \\
\tau_{2}= & 2 c_{1} \cos \alpha\left[\sin \theta \sin \psi \cos \theta^{*}-(\cos \theta \cos \phi \sin \psi+\sin \phi \cos \psi) \sin \theta^{*} \cos \phi^{*}\right. \\
& \left.+(-\cos \theta \sin \phi \sin \psi+\cos \phi \cos \psi) \sin \theta^{*} \sin \phi^{*}\right]
\end{aligned}
$$

The expressions for the components $\tau_{1}^{*}$ and $\tau_{2}^{*}$ of the torque $\tau^{*}$ over the internal ellipsoid are like above, provided the exchanges $\theta \leftrightarrow \theta^{*}, \phi \leftrightarrow \phi^{*}, \psi \leftrightarrow \psi^{*}$ are made. In the same way, we also need the expression for $\cos \alpha$

$$
\cos \alpha=\sin \theta \sin \theta^{*} \cos \left(\phi-\phi^{*}\right)+\cos \theta \cos \theta^{*} .
$$

After substituting equations (14)-(18) into equations (8), (9), (11), (12) and considering that we are dealing with real quantities we can eliminate $\psi, \psi^{*}, \dot{\psi}, \dot{\psi}^{*}$, getting the following set of four second-order differential equations

$$
\begin{aligned}
& \ddot{\phi} \sin \theta-\frac{C}{A} \omega_{3} \dot{\theta}+2 \dot{\theta} \dot{\phi} \cos \theta-2 \frac{c_{1}}{A} \cos \alpha \sin \theta^{*} \sin \left(\phi^{*}-\phi\right)=0 \\
& \ddot{\theta}-\dot{\phi}^{2} \sin \theta \cos \theta+\frac{C}{A} \omega_{3} \dot{\phi} \sin \theta+2 \frac{c_{1}}{A} \cos \alpha\left[\sin \theta \cos \theta^{*}-\cos \theta \sin \theta^{*} \cos \left(\phi-\phi^{*}\right)\right]=0 \\
& \ddot{\phi}^{*} \sin \theta^{*}-\frac{C^{*}}{A^{*}} \omega_{3^{*}}^{*} \dot{\theta}^{*}+2 \dot{\theta}^{*} \dot{\phi}^{*} \cos \theta^{*}-2 \frac{c_{1}}{A^{*}} \cos \alpha \sin \theta \sin \left(\phi-\phi^{*}\right)=0 \\
& \ddot{\theta}^{*}-\dot{\phi}^{* 2} \sin \theta^{*} \cos \theta^{*}+\frac{C^{*}}{A^{*}} \omega_{3^{*}}^{*} \dot{\phi}^{*} \sin \theta^{*}+2 \frac{c_{1}}{A^{*}} \cos \alpha\left[\sin \theta^{*} \cos \theta\right. \\
& \left.\quad-\cos \theta^{*} \sin \theta \cos \left(\phi-\phi^{*}\right)\right]=0 .
\end{aligned}
$$

As a test for these equations we consider the case where $c_{1}=0$, what means that the ellipsoids are not interacting and therefore free precession occurs. It is known (see $e$. g. Symon [15]) in this case that for each ellipsoid both polar principal symmetry axis (3-axis) and the rotation axis, which contains the angular velocity vector $\omega$, have precession around the conserved angular momentum vector $\mathbf{L}$ forming the so called body cone and space cone, with semi-angles $\alpha_{b}$ and $\alpha_{s}$, respectively (see Fig. 3). Notice that the 3 -axis, $\boldsymbol{\omega}$ and $\mathbf{L}$ are always in the same plane. The constant angular velocity of such a precession around fixed $\mathbf{L}$ is

$$
\dot{\phi}^{\prime}=\beta \frac{\sin \alpha_{b}}{\sin \alpha_{s}} \omega_{3}=\frac{C}{A \cos \theta^{\prime}} \omega_{3}
$$

with

$$
\theta^{\prime}=\alpha_{b}-\alpha_{s}
$$




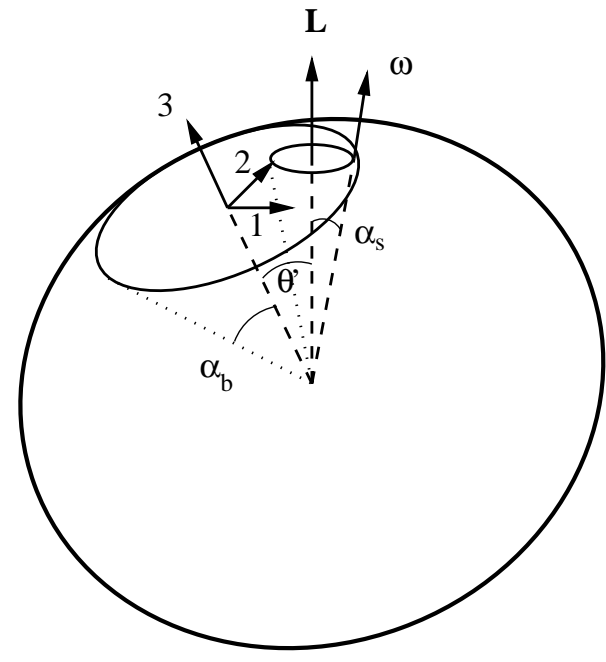

FIG. 3: Space and body cones generated by the Eulerian (free) precession. The axes 1, 2 (equatorial principal axes) and 3 (polar principal axis) are fixed in the oblate ellipsoid and follow its motion. The angular momentum $\mathbf{L}$ defines a fixed direction in space while the angular velocity $\boldsymbol{\omega}$ gives the instantaneous rotation axis. being the constant angle that gives the orientation of the principal 3-axis with respect to fixed $\mathbf{L}$, and

$$
\begin{aligned}
& \tan \alpha_{b}=\frac{\sqrt{\omega_{1}^{2}+\omega_{2}^{2}}}{\omega_{3}} \\
& \cos \alpha_{s}=\frac{1+\beta \cos ^{2} \alpha_{b}}{\sqrt{1+(2+\beta) \beta \cos ^{2} \alpha_{b}}} \\
& \beta=\frac{C}{A}-1 .
\end{aligned}
$$

However, with two ellipsoids, we have a more general situation where the fixed reference frame $\mathrm{Z}$-axis is taken to be in the direction of the total angular momentum $\mathbf{L}_{t}$, which is conserved if external torques are negligible, and not of the Earth angular momentum $\mathbf{L}$. Therefore we define a reference frame $\mathrm{xyz}$, such that $\mathrm{z}$ is in the direction of $\mathbf{L}$, oriented with respect to XYZ through the Euler angles $\delta_{\theta}, \delta_{\phi}, \delta_{\psi}$ (see Fig. 4). While the principal 3-axis has precession around $\mathrm{z}$-axis with constant angular velocity $\dot{\phi}^{\prime}$ and angular amplitude $\theta^{\prime}$, the 3 -axis has motion described in terms of the Euler angles $\theta$, $\phi$ with respect to the frame XYZ as

$$
\begin{aligned}
& \cos \theta=\sin \theta^{\prime}\left[\sin (\dot{\phi} t+\varepsilon) \sin \delta_{\theta} \sin \delta_{\psi}-\cos (\dot{\phi} t+\varepsilon) \sin \delta_{\theta} \cos \delta_{\psi}\right]+\cos \theta^{\prime} \cos \delta_{\theta} \\
& \tan \phi=-\frac{\sin \theta^{\prime}\left[B_{1} \sin (\dot{\phi} t+\varepsilon)+B_{2} \cos (\dot{\phi} t+\varepsilon)\right]+\cos \theta^{\prime} \sin \delta_{\theta} \sin \delta_{\phi}}{\sin \theta^{\prime}\left[B_{3} \sin (\dot{\phi} t+\varepsilon)+B_{4} \cos (\dot{\phi} t+\varepsilon)\right]-\cos \theta^{\prime} \sin \delta_{\theta} \cos \delta_{\phi}}
\end{aligned}
$$

with $\varepsilon$ being an arbitrary phase and

$$
\begin{aligned}
& B_{1}=\cos \delta_{\phi} \cos \delta_{\psi}-\cos \delta_{\theta} \sin \delta_{\phi} \sin \delta_{\psi} \\
& B_{2}=\cos \delta_{\phi} \sin \delta_{\psi}+\cos \delta_{\theta} \sin \delta_{\phi} \cos \delta_{\psi} \\
& B_{3}=\sin \delta_{\phi} \cos \delta_{\psi}+\cos \delta_{\theta} \cos \delta_{\phi} \sin \delta_{\psi} \\
& B_{4}=\sin \delta_{\phi} \sin \delta_{\psi}-\cos \delta_{\theta} \cos \delta_{\phi} \cos \delta_{\psi}
\end{aligned}
$$

It can be verified using an algebraic calculation software that $\theta$ and $\phi$ given by equations (29) and (30) satisfy equations (20) and (21) with $c_{1}=0$, what means that the latter equations describe correctly the precessional motion around an axis oriented arbitrarily in space.

Before proceeding to the solution of equations (20)-(23) let us consider the elasticity of the Earth. This was first taken into account by Love [1] and Larmor [2] because the predicted free precession period was of 305 days while the observed value was of around 435 days. The correction due to Love introduces products of inertia $C_{13}$ and $C_{23}$ in Euler equations (8)-(9) for the Earth (see Kaula [16]) which become

$$
\begin{aligned}
& A \dot{\omega}_{1}+(C-A) \omega_{2} \omega_{3}-C_{23} \omega_{3}^{2}+\dot{C}_{13} \omega_{3}=\tau_{1} \\
& A \dot{\omega}_{2}-(C-A) \omega_{1} \omega_{3}+C_{13} \omega_{3}^{2}+\dot{C}_{23} \omega_{3}=\tau_{2}
\end{aligned}
$$

with

$$
\begin{aligned}
& C_{13}=\frac{k_{2} R_{E}^{5} \omega_{3}}{3 G} \omega_{1} \\
& C_{23}=\frac{k_{2} R_{E}^{5} \omega_{3}}{3 G} \omega_{2}
\end{aligned}
$$

where $R_{E}$ is the Earth mean radius and $k_{2}$ is the so called Love number. Therefore we obtain as a result

$$
\begin{aligned}
& \left(A+\frac{k_{2} R_{E}^{5} \omega_{3}^{2}}{3 G}\right) \dot{\omega}_{1}+\left(C-A-\frac{k_{2} R_{E}^{5} \omega_{3}^{2}}{3 G}\right) \omega_{2} \omega_{3}=\tau(39) \\
& \left.\left(A+\frac{k_{2} R_{E}^{5} \omega_{3}^{2}}{3 G}\right) \dot{\omega}_{2}-\left(C-A-\frac{k_{2} R_{E}^{5} \omega_{3}^{2}}{3 G}\right) \omega_{1} \omega_{3}=\tau_{6} 40\right)
\end{aligned}
$$

By comparing this with equations (8)-(9) we see that the overall effect upon the last ones by the introduction of the elasticity of the Earth is to promote the transformation

$$
A \rightarrow A+\frac{k_{2} R_{E}^{5} \omega_{3}^{2}}{3 G} .
$$

The Love number $k_{2}$ is left as one of the free parameters of this model.

We solve numerically the set of four second-order differential equations given by (20)-(23), after performing the transformation given by equation (41), through the Runge-KuttaFehlberg (RKF) method. The time step upper limit is 1 solar 


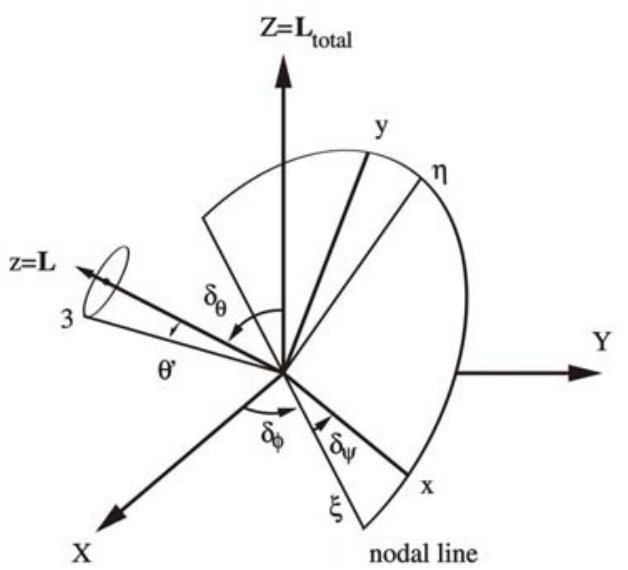

FIG. 4: Free precession around an arbitrary axis z. The xyz-frame is oriented with respect to the XYZ-frame, which is fixed in space, through the Euler angles $\delta_{\theta}, \delta_{\phi}, \delta_{\psi}$.

day $/ 1000=86.4 \mathrm{~s} ;$ changing this limit to 1 solar day $/ 10000 \mathrm{did}$ not cause meaningful modifications in the results. We have 11 free parameters, $i$. e., the Love number $k_{2}$, the momenta of inertia $A^{*}, C^{*}$ of the internal ellipsoid, and the following that establish the initial conditions: the components $\omega_{1^{*}}^{*}, \omega_{2^{*}}^{*}, \omega_{3^{*}}^{*}$ (recall that $\omega_{3^{*}}^{*}$ is constant) of the angular velocity $\omega^{*}$ in the principal axes $1^{*} 2^{*} 3^{*}$ of the internal ellipsoid, the Euler angles $\theta_{12}=\alpha, \phi_{12}, \psi_{12}$ that give the spatial orientation of the $1^{*} 2^{*} 3^{*}$ frame with respect to the 123 frame, and the angular velocities $\dot{\phi}$ and $\dot{\phi}^{*}$. On the other hand, from equations (14) and (15) we have

$$
\dot{\theta}= \pm \sqrt{\omega_{1}^{2}+\omega_{2}^{2}-\dot{\phi}^{2} \sin ^{2} \theta}
$$

from which we can determine the initial value of $\dot{\theta}$ unless by its sign. In an analogous way we have

$$
\dot{\theta}^{*}= \pm \sqrt{\omega_{1^{*}}^{* 2}+\omega_{2^{*}}^{* 2}-\dot{\phi}^{* 2} \sin ^{2} \theta^{*}} .
$$

From equations (42)-(43) we can also find bounds for the initial guesses of $\dot{\phi}$ and $\dot{\phi}^{*}$

$$
\begin{aligned}
& -\frac{\sqrt{\omega_{1}^{2}+\omega_{2}^{2}}}{|\sin \theta|} \leq \dot{\phi} \leq \frac{\sqrt{\omega_{1}^{2}+\omega_{2}^{2}}}{|\sin \theta|} \\
& -\frac{\sqrt{\omega_{1^{*}}^{* 2}+\omega_{2^{*}}^{* 2}}}{\left|\sin \theta^{*}\right|} \leq \dot{\phi}^{*} \leq \frac{\sqrt{\omega_{1^{*}}^{* 2}+\omega_{2^{*}}^{* 2}}}{\left|\sin \theta^{*}\right|} .
\end{aligned}
$$

Besides, from energy conservation of the Earth-ellipsoid system, we have the following relation to be satisfied by the initial guesses of $\omega_{1^{*}}^{*}$ and $\omega_{2^{*}}^{*}$

$$
\begin{aligned}
& \omega_{1^{*}}^{* 2}+\omega_{2^{*}}^{* 2} \geq \frac{c_{1}}{A}[\cos (2 \alpha)-1]-\frac{A}{A^{*}}\left(\omega_{1}^{2}+\omega_{2}^{2}\right)-\frac{2 \omega_{3}}{A^{*}} \times \\
& \times\left(C_{13} \omega_{1}+C_{23} \omega_{2}\right)
\end{aligned}
$$

where $\alpha$ is the initial guess for the angle between the axes 3 and $3^{*}$, and $\omega_{1}$ and $\omega_{2}$ are the initial components of the Earth angular velocity $\boldsymbol{\omega}$ in the axes 1 and 2, respectively.
Höpfner [11, 12] has managed to filter the Chandler wobble (CW) and the annual wobble (AW) as major components from the data related to the Earth polar motion of its rotation axis around the polar principal axis. These data have being accumulated for more than 100 years and a compilation of them is published periodically by Gross [17] from NASA-JPL. We use a relation between the polar motion, described as the two angles $\widehat{P M X}$ and $\widehat{P M Y}$, and the orientation of the Earth rotation axis, described by the angles $\theta_{\omega}$ and $\phi_{\omega}$ (see Fig. 5). As usual, the 1-axis is towards the Greenwich meridian and the 2-axis is towards the $90^{\circ} \mathrm{E}$ meridian. The angles $\theta_{\omega}$ and $\phi_{\omega}$ can be calculated from

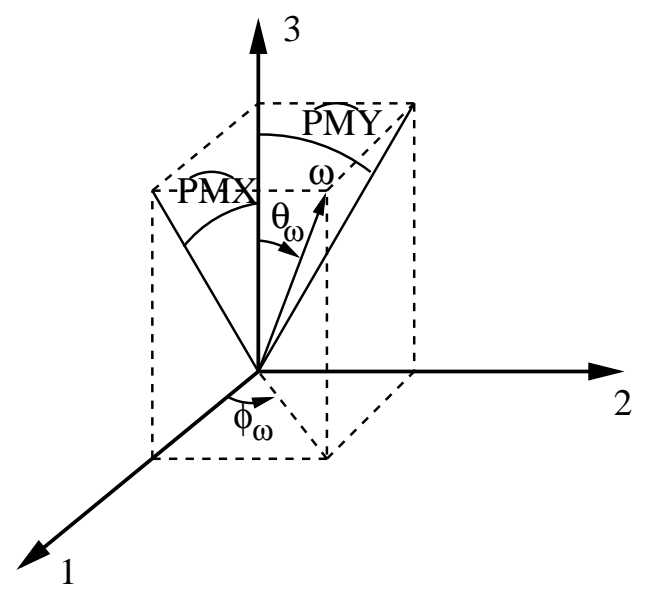

FIG. 5: Definition of the angles $\widehat{P M X}$ and $\widehat{P M Y}$ which describe the Chandler wobble motion. The origin of the 123-frame is at the Earth center. Axes 1 and 2 are towards the Greenwich meridian and $90^{\circ} \mathrm{E}$ meridian, respectively. The 3 -axis is the polar principal axis. The angular velocity $\boldsymbol{\omega}$ is also shown as well as its spherical coordinates $\theta_{\omega}$ and $\phi_{\omega}$.

$$
\begin{aligned}
\tan \theta_{\omega} & = \pm \sqrt{\tan ^{2} \widehat{P M X}+\tan ^{2} \widehat{P M Y}} \\
\tan \phi_{\omega} & =\frac{\tan \widehat{P M Y}}{\tan \widehat{P M X}} .
\end{aligned}
$$

Therefore by knowing $\widehat{P M X}$ and $\widehat{P M Y}$ at the start of a Chandler wobble, taken from Höpfner [11] calculations, and by knowing the value of the length-of-day (LOD) at that moment, taken from Gross [17] data, we can calculate the initial values of $\omega_{1}$ and $\omega_{2}$, which are necessary in equations (42), (44) and (46), and the value of $\omega_{3}$, which is considered as constant during the motion. Furthermore, we can calculate then the initial components of the Earth angular momentum $\mathbf{L}$ in the direction of the axes 123 as

$$
\begin{aligned}
& L_{1}=A \omega_{1}+C_{13} \omega_{3} \\
& L_{2}=A \omega_{2}+C_{23} \omega_{3} \\
& L_{3}=C \omega_{3}+C_{13} \omega_{1}+C_{23} \omega_{2} .
\end{aligned}
$$

Once we have guessed the initial values of $\omega_{1^{*}}^{*}, \omega_{2^{*}}^{*}, \omega_{3^{*}}^{*}$, we can also calculate the initial values of the components of $\mathbf{L}^{*}$ in 
the axes $1 * 2 * 3 *$ by

$$
\begin{aligned}
& L_{1^{*}}^{*}=A^{*} \omega_{1^{*}}^{*} \\
& L_{2^{*}}^{*}=A^{*} \omega_{2^{*}}^{*} \\
& L_{3^{*}}^{*}=C^{*} \omega_{3^{*}}^{*} .
\end{aligned}
$$

With these quantities available we can calculate the components of the total angular momentum $\mathbf{L}_{t}$ in the axes 123

$$
\begin{gathered}
L_{t 1}=L_{1}+L_{1}^{*}=L_{1}+L_{1^{*}}^{*}\left(\cos \phi_{12} \cos \psi_{12}-\cos \alpha \sin \phi_{12} \sin \psi_{12}\right) \\
-L_{2^{*}}^{*}\left(\cos \phi_{12} \sin \psi_{12}+\cos \alpha \sin \phi_{12} \cos \psi_{12}\right)+L_{3^{*}}^{*} \sin \alpha \sin \phi_{12} \\
\begin{aligned}
& L_{t 2}=L_{2}+L_{2}^{*}=L_{2}+L_{1^{*}}^{*}\left(\sin \phi_{12} \cos \psi_{12}+\cos \alpha \cos \phi_{12} \sin \psi_{12}\right) \\
&+ L_{2^{*}}^{*}\left(-\sin \phi_{12} \sin \psi_{12}+\cos \alpha \cos \phi_{12} \cos \psi_{12}\right)-L_{3^{*}}^{*} \sin \alpha \cos \phi_{12} \\
& L_{t 3}=L_{3}+L_{3}^{*}=L_{3}+L_{1^{*}}^{*} \sin \alpha \sin \psi_{12}+L_{2^{*}}^{*} \sin \alpha \cos \psi_{12}+L_{3^{*}}^{*} \cos \alpha .
\end{aligned}
\end{gathered}
$$

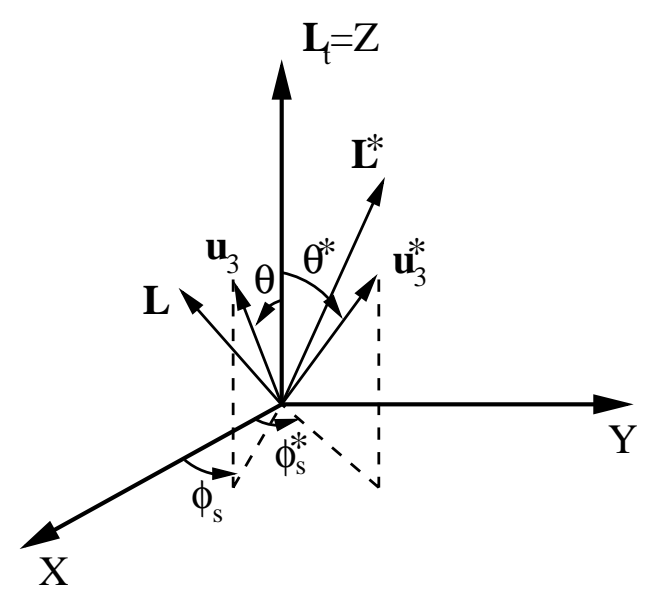

FIG. 6: Definition of the frame XYZ fixed in space which is convenient to describe both interacting ellipsoids motion. The $\mathrm{Z}$-axis coincides with the direction of the total angular momentum $\mathbf{L}_{t}$. The XZ-plane is the same plane formed by the $\mathbf{L}$ (Earth) and $\mathbf{L}^{*}$ (ellipsoid) angular momenta. The unit vectors $\mathbf{u}_{3}$ and $\mathbf{u}_{3}^{*}$, which are in the direction of the polar principal axes 3 and $3^{*}$, are also shown as well as their spherical coordinates $\theta, \phi_{s}, \theta^{*}, \phi_{s}^{*}$.

Here, $\alpha=\theta_{12}, \phi_{12}$ and $\psi_{12}$ are the initial guesses for the Euler angles that describe the $1^{*} 2^{*} 3^{*}$ reference frame with respect to the 123 frame. Since we define the $\mathrm{Z}$-axis of the fixed reference frame in the same direction of $\mathbf{L}_{t}$ (see Fig. 6), the initial value of the Euler angle $\theta$, that is necessary for the solution of equations (20)-(23), is obtained from

$$
\cos \theta=\frac{\mathbf{L}_{t} \cdot \mathbf{u}_{3}}{L_{t}}=\frac{L_{t 3}}{\sqrt{L_{t 1}^{2}+L_{t 2}^{2}+L_{t 3}^{2}}}
$$

where $\mathbf{u}_{3}$ is a unit vector in the direction of the 3-axis. In order to calculate the initial value of $\phi$ we construct the Y-axis as orthogonal to the $\mathbf{L}^{*} \mathbf{L}$-plane (Fig. 6), taking the initial values of such vectors, and the $\mathrm{X}$-axis orthogonal to the YZ-plane, as

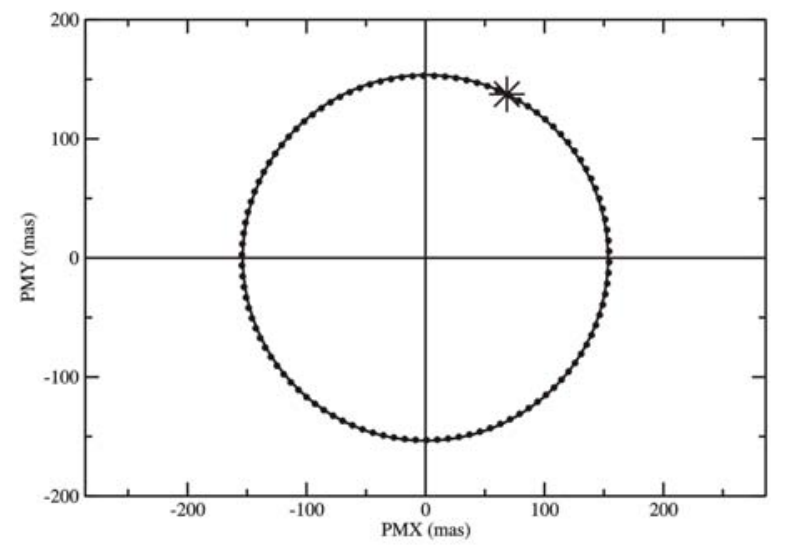

FIG. 7: Calculated (line) and predicted (dots) Chandler wobble prograde (counter-clockwise) motion in the interval 44330-44755 (in modified Julian date - MJD) or 01/APR/1980-31/MAY/1981. The asterisk shows the position of the motion start point. $\widehat{P M X}$ and $\widehat{P M Y}$ are given in milli-arcsec (mas).

usual. Then

$$
\cos \phi=-\frac{u_{3 Y}}{u_{3 X Y}}=\frac{L_{t 2} L_{1}-L_{t 1} L_{2}}{\left|\mathbf{L} \times \mathbf{L}_{t}\right| \sin \theta}
$$

where $u_{3 Y}$ and $u_{3 X Y}$ are the projections of $\mathbf{u}_{3}$ in the Y-axis and in the XY-plane, respectively. In order to determine $\phi$ completely we have also to express

$$
\sin \phi=\frac{u_{3 X}}{u_{3 X Y}}=\frac{L_{t 2}\left(L_{t 2} L_{3}-L_{t 3} L_{2}\right)-L_{t 1}\left(L_{t 3} L_{1}-L_{t 1} L_{3}\right)}{L_{t}\left|\mathbf{L} \times \mathbf{L}_{t}\right| \sin \theta} .
$$

In an analogous way we have the projections of $\mathbf{L}_{t}$ in the axes $1 * 2 * 3 *$ 


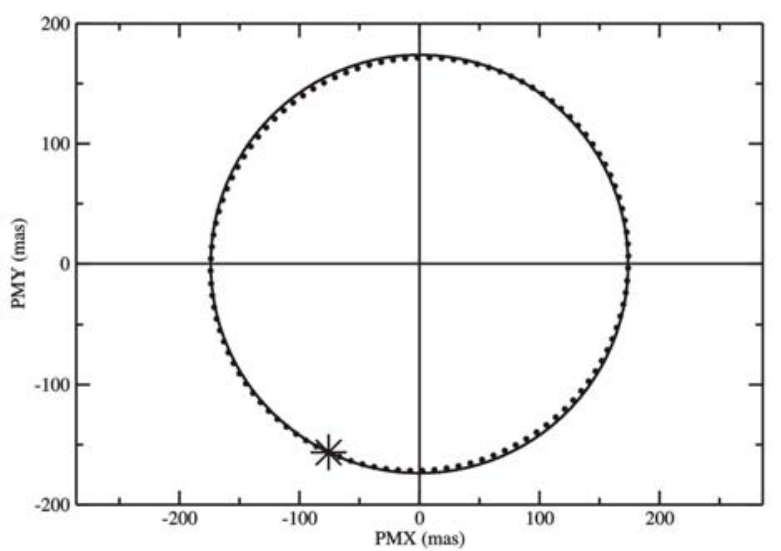

FIG. 8: Same as in Fig. 7, for the interval 44970-45403 (MJD) or 01/JAN/1982-10/MAR/1983.

$$
\begin{aligned}
& L_{t 1^{*}}=L_{1^{*}}^{*}+L_{1^{*}}=L_{1^{*}}^{*}+L_{1}\left(\cos \phi_{12} \cos \psi_{12}-\cos \alpha \sin \phi_{12} \sin \psi_{12}\right) \\
& +L_{2}\left(\sin \phi_{12} \cos \psi_{12}+\cos \alpha \cos \phi_{12} \sin \psi_{12}\right)+L_{3} \sin \alpha \sin \psi_{12} \\
& L_{t 2^{*}}=L_{2^{*}}^{*}+L_{2^{*}}=L_{2^{*}}^{*}-L_{1}\left(\cos \phi_{12} \sin \psi_{12}+\cos \alpha \sin \phi_{12} \cos \psi_{12}\right) \\
& +L_{2}\left(-\sin \phi_{12} \sin \psi_{12}+\cos \alpha \cos \phi_{12} \cos \psi_{12}\right)+L_{3} \sin \alpha \cos \psi_{12} \\
& L_{t 3^{*}}=L_{3^{*}}^{*}+L_{3^{*}}=L_{3^{*}}^{*}+L_{1} \sin \alpha \sin \phi_{12}-L_{2} \sin \alpha \cos \phi_{12}+L_{3} \cos \alpha \text {. }
\end{aligned}
$$

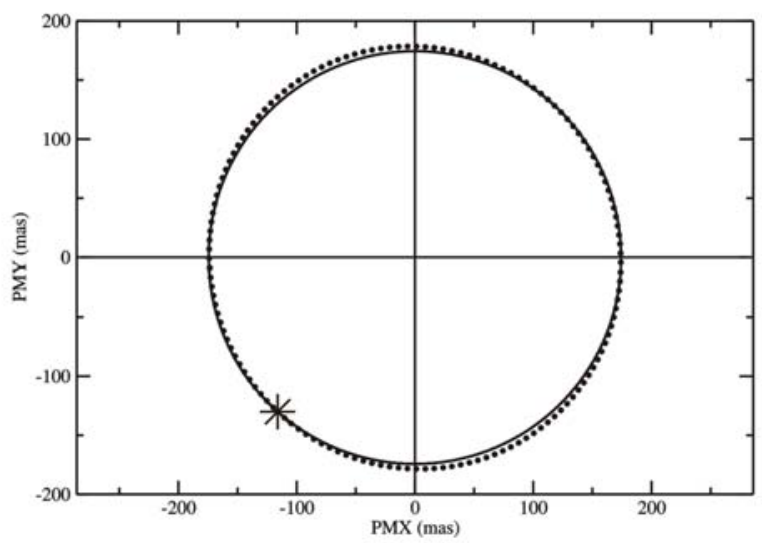

FIG. 9: Same as in Fig. 7, for the interval 50170-50604 (MJD) or 28/MAR/1996-05/JUN/1997.

and the initial values for the Euler angles $\theta^{*}$ and $\phi^{*}$ are obtained from

$$
\begin{aligned}
\cos \theta^{*} & =\frac{\mathbf{L}_{t} \cdot \mathbf{u}_{3}^{*}}{L_{t}}=\frac{L_{t 3^{*}}}{\sqrt{L_{t 1^{*}}^{2}+L_{t 2^{*}}^{2}+L_{t 3^{*}}^{2}}} \\
\cos \phi^{*} & =-\frac{u_{3 Y}^{*}}{u_{3 X Y}^{*}}=\frac{L_{t 1^{*}} L_{2^{*}}^{*}-L_{t 2^{*}} L_{1^{*}}^{*}}{\left|\mathbf{L}^{*} \times \mathbf{L}_{t}\right| \sin \theta^{*}} \\
\sin \phi^{*} & = \\
\frac{u_{3 X}^{*}}{u_{3 X Y}^{*}} & =\frac{L_{t 2^{*}}\left(L_{t 3^{*}} L_{2^{*}}^{*}-L_{t 2^{*}} L_{3^{*}}^{*}\right)-L_{t 1^{*}}\left(L_{t 1^{*}} L_{3^{*}}^{*}-L_{t 3^{*}} L_{1^{*}}^{*}\right)}{L_{t}\left|\mathbf{L}^{*} \times \mathbf{L}_{t}\right| \sin \theta^{*}} .
\end{aligned}
$$

While integrating equations (20)-(23) we can calculate the CW motion through (see Fig. 5)

$$
\begin{aligned}
& \tan \widehat{P M X}=\frac{\omega_{1}}{\omega_{3}} \\
& \tan \widehat{P M Y}=\frac{\omega_{2}}{\omega_{3}}
\end{aligned}
$$

with $\omega_{1}$ and $\omega_{2}$ given by equations (14) and (15). The necessary values of $\psi$ are obtained step-by-step through numerical integration of equation (16). 


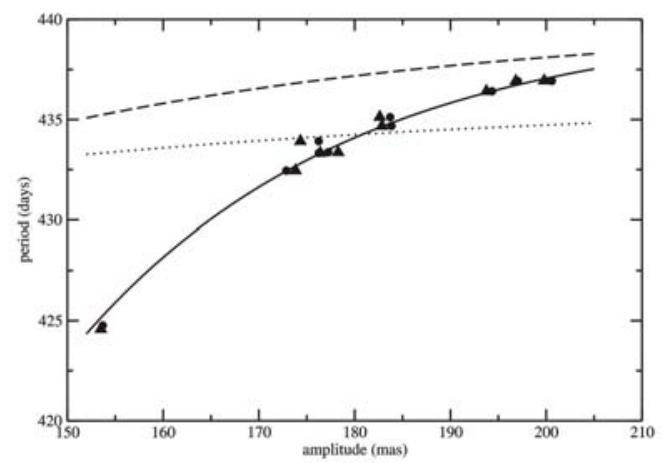

FIG. 10: Relation between CW period (in days) and average amplitude (in mas). Circles represent results due to Höpfner [11] while triangles show our results. The full line fits the last points. As a reference, dashed and dotted lines represent fits for the epochs 1912-1928 and 1936-1948 respectively.

\section{RESULTS AND DISCUSSION}

With the aim of getting 10 unknown parameters of our model (all but $k_{2}$ ) we find that it is enough to establish the following conditions: 1) reproduce the Chandler component of the Earth rotation axis position predicted by Höpfner [11] at the start of a wobble; 2) minimize the length of the prograde (counter-clockwise) trajectory followed during that wobble motion. The second condition is necessary in order to avoid intrusive nutations that might emerge accompanying the precessional motion. This means that we minimize, for a given initial guess of $k_{2}$, the sum of: a) the squared distance from the model calculed Earth rotation axis position $(\widehat{P M X}, \widehat{P M Y})$ at the start of the wobble and the one predicted by Höpfner; b) the wobble trajectory length in the plane $(\widehat{P M X}, \widehat{P M Y})$ obtained by numerical integration. For this minimization process we used the POWELL routine from the Numerical Recipes package (Press et al. [18]). However the generalized simulated annealing (GSA) code (Mundim and Tsallis [19], Dall'Igna Junior et al. [20]) has proved to be very effective in a first step run in order to prevent getting stuck in local minima. The 11 th parameter, $k_{2}$, is then found by reproducing the Chandler component of the Earth rotation axis position at the end of the wobble, with reference to Höpfner's prediction, so that $k_{2}$ is responsible for the adjustment of a particular wobble period. We show in Figs. 7-9 some of our results for the CW in three different epochs, in comparison with the results due to Höpfner [11], which are represented by dots. The first one, for which $\omega_{3^{*}}^{*}$ is negative, has the typical quality of providing reasonable reproduction of the motion. The next Figures show the two exceptions to this rule, which tend to present almost circular trajectories instead of, in those epochs, the slightly elliptical ones due to Höpfner. This may be caused by the above mentioned criteria - mainly condition 2) - adopted in the fit process. We also show in Table I the corresponding values for the 11 parameters (with respective numerical uncertainties shown in Table II), while in Table III we have the initial values of $\dot{\theta}$ and $\dot{\theta}^{*}$ calculated from equations (42) and (43) with appropriate signs and the corresponding values of the torque strength $c_{1}$. As expected - see comments after equation (7) $-\alpha$ turns out to be close to $90^{\circ}$. On the other hand, parameters $\omega_{3^{*}}^{*}$ (the main angular velocity component of the ellipsoid), $A^{*}, C^{*}$ and $k_{2}$ present oscillations when examining the respective columns in the Table I, being more noticeable for the 44330-44755 interval. For $k_{2}$, since it is connected to the period and that particular wobble has a short one - see Table IV for a comparison between the presently calculated periods and the ones due to Höpfner which they shall reproduce - this is not surprising although undesirable. Höpfner $[11,12]$, like other authors (see $e$. g. Liu et al. [21], Wang [22]), found variable CW periods but this is not a clearly solved question since some investigators have another opinion (see e. g. Vicente and Wilson [23], Jochmann [24], Liao and Zhou [25], Guo et al. [26]). For the sake of comparison, values of $k_{2}$ obtained by some other authors are 0.284 (Kaula [16]), 0.30088 (model 1066A of Gilbert and Dziewonski [27]) which are not far from what we got with the exception of the 44330-44755 case.

By comparing the values of $C^{*}$ with momenta of inertia of natural satellites present in the solar system we find that they range from about Miranda's (Uranus) to Charon's (Pluto). Since we are able only to calculate the DM ellipsoid principal momenta of inertia, which are related to the ellipsoid mass $M^{*}$ and their equatorial and polar radii $a^{*}$ and $c^{*}$ through $A^{*}=M^{*}\left(a^{* 2}+c^{* 2}\right) / 5$ and $C^{*}=2 M^{*} a^{* 2} / 5$, we cannot establish the ellipsoid mass uniquely, and in consequence, the fraction of DM present in the Earth is not predicted. Notice that, for an oblate ellipsoid, we have $c^{*} \leq a^{*}$ and, therefore, the relation $C^{*} / 2 \leq A^{*} \leq C^{*}$ holds and it was required to be satisfied in our numerical procedure. Just to have an idea of the order of magnitude of $M^{*}$, let us suppose that $a^{*} \approx 2350 \mathrm{~km}, i$. $e$., the ellipsoid has the average radius of the outer core of the Earth. From the values of $C^{*}$ in Table I, this implies that the fraction of DM present in the Earth would lie in the range $6 \times 10^{-7}$ to $9 \times 10^{-5}$. We emphasize that, according to the model, the Earth motion and its gravitational interaction with other bodies have already incorporated the presence of DM through what has been known effectively as "Earth mass", so that the outcome of its presence might be more easily observed through the Earth wobble.

Following the hypothesis of changing $\mathrm{CW}$ periods we present in Fig. 10 the correlation between period and average amplitude. We show Höpfner's results [11] and ours. The last ones are fitted by the full line, which has the form: period (in days) $=440-3101.47 \times \exp [-0.0347946 \times$ amplitude (in mas)]. As a reference we also present the fits due to Liu et al [21] related to the epochs 1912-1928 and 1936-1948. The correlation clearly varies with time.

One might argue that the parameter fluctuations in Table I indicate that this model is not sound. However those fluctuations may be caused by the model simplistic - although convenient at this stage - approach that we are considering the DM ellipsoid the only source responsible for the complexity of Chandler wobble, what obviously is not true. Therefore, the predicted $A^{*}$ and $C^{*}$ dark matter ellipsoid parameters should be considered only as upper limit values.

Another point that could be raised against the model is that it is just emulating one or more of the attributed causes for $\mathrm{CW}$ - as listed in the Introduction - and, in consequence, the DM ellipsoid existence is not real. However, it can be shown that externally to the DM ellipsoid, whose rotation axis is almost orthogonal to the Earth's, it generates a non-isotropic, time 
TABLE I: Results for the 11 unknown parameters in several epochs: initial values for the angular velocities $\dot{\phi}$ and $\dot{\phi}^{*}$ (in rad/s), for the components $\omega_{1^{*}}^{*}, \omega_{2^{*}}^{*}, \omega_{3^{*}}^{*}$ (in rad/s) of angular velocity $\omega^{*}$, for the Euler angles $\alpha=\theta_{12}, \phi_{12}, \psi_{12}$ (in degrees); values of the internal ellipsoid principal momenta of inertia $A^{*}, C^{*}$ (in $\mathrm{kg} \cdot \mathrm{m}^{2}$ ) and Love number $k_{2}$.

\begin{tabular}{|c|c|c|c|c|c|c|c|c|c|c|c|}
\hline interval & $\dot{\phi}$ & $\dot{\phi}^{*}$ & $\omega_{1^{*}}^{*}$ & $\omega_{2^{*}}^{*}$ & $\omega_{3^{*}}^{*}$ & $\phi_{12}$ & $\psi_{12}$ & $\alpha$ & $A^{*}$ & $C^{*}$ & $k_{2}$ \\
\hline $44330-44755$ & $1.386 \mathrm{E}-7$ & $3.087 \mathrm{E}-20$ & $9.907 \mathrm{E}-12$ & $4.140 \mathrm{E}-10$ & $-1.592 \mathrm{E}-4$ & 358.849 & 353.219 & 90.001 & $6.334 \mathrm{E}+32$ & $1.264 \mathrm{E}+33$ & 0.2672 \\
\hline $44970-45403$ & $-2.704 \mathrm{E}-5$ & $-9.685 \mathrm{E}-7$ & $-9.789 \mathrm{E}-7$ & $-2.088 \mathrm{E}-9$ & $4.952 \mathrm{E}-4$ & 173.698 & 337.477 & 89.911 & $2.399 \mathrm{E}+31$ & $3.334 \mathrm{E}+31$ & 0.2801 \\
\hline $45700-46135$ & $5.711 \mathrm{E}-5$ & $-4.203 \mathrm{E}-6$ & $-4.262 \mathrm{E}-6$ & $-2.128 \mathrm{E}-9$ & $6.853 \mathrm{E}-4$ & 359.988 & 359.999 & 89.847 & $1.079 \mathrm{E}+31$ & $1.125 \mathrm{E}+31$ & 0.2835 \\
\hline $46340-46773$ & $-7.419 \mathrm{E}-5$ & $1.823 \mathrm{E}-6$ & $3.935 \mathrm{E}-6$ & $-2.410 \mathrm{E}-9$ & $1.089 \mathrm{E}-3$ & 187.700 & 350.276 & 89.849 & $7.429 \mathrm{E}+30$ & $7.772 \mathrm{E}+30$ & 0.2815 \\
\hline $46977-47410$ & $7.205 \mathrm{E}-5$ & $-4.490 \mathrm{E}-6$ & $-4.542 \mathrm{E}-6$ & $-2.128 \mathrm{E}-9$ & $7.455 \mathrm{E}-4$ & 359.988 & 359.999 & 89.857 & $1.168 \mathrm{E}+31$ & $1.211 \mathrm{E}+31$ & 0.2815 \\
\hline $47640-48075$ & $-3.769 \mathrm{E}-5$ & $2.098 \mathrm{E}-6$ & $3.572 \mathrm{E}-6$ & $-2.410 \mathrm{E}-9$ & $7.727 \mathrm{E}-4$ & 109.191 & 353.481 & 89.832 & $7.280 \mathrm{E}+30$ & $7.548 \mathrm{E}+30$ & 0.2842 \\
\hline $48260-48696$ & $5.317 \mathrm{E}-6$ & $-1.861 \mathrm{E}-7$ & $-1.862 \mathrm{E}-7$ & $-2.128 \mathrm{E}-9$ & $3.338 \mathrm{E}-3$ & 222.469 & 359.999 & 90.047 & $1.947 \mathrm{E}+31$ & $2.125 \mathrm{E}+31$ & 0.2861 \\
\hline $48900-49336$ & $-2.505 \mathrm{E}-5$ & $-1.142 \mathrm{E}-6$ & $-1.142 \mathrm{E}-6$ & $-2.088 \mathrm{E}-9$ & $5.632 \mathrm{E}-4$ & 346.867 & 332.210 & 89.841 & $1.731 \mathrm{E}+31$ & $1.903 \mathrm{E}+31$ & 0.2869 \\
\hline $49630-50066$ & $4.133 \mathrm{E}-5$ & $-6.759 \mathrm{E}-6$ & $-6.759 \mathrm{E}-6$ & $-2.128 \mathrm{E}-9$ & $7.390 \mathrm{E}-4$ & 359.988 & 359.999 & 89.873 & $9.482 \mathrm{E}+30$ & $9.710 \mathrm{E}+30$ & 0.2869 \\
\hline $50170-50604$ & $-3.317 \mathrm{E}-5$ & $-6.301 \mathrm{E}-7$ & $-6.301 \mathrm{E}-7$ & $-2.088 \mathrm{E}-9$ & $5.230 \mathrm{E}-4$ & 247.397 & 333.076 & 89.877 & $8.287 \mathrm{E}+30$ & $9.587 \mathrm{E}+30$ & 0.2824 \\
\hline
\end{tabular}

TABLE II: Numerical uncertainty estimates (in \%) of the parameters shown in Table I.

\begin{tabular}{|c|c|c|c|c|c|c|c|c|c|c|c|}
\hline interval & $\left|\frac{\Delta \dot{\phi}}{\dot{\phi}}\right|$ & $\left|\frac{\Delta \dot{\phi}^{*}}{\dot{\phi}^{*}}\right|$ & $\left|\frac{\Delta \omega_{1 *}^{*}}{\omega_{1 *}^{*}}\right|$ & $\left|\frac{\Delta \omega_{2^{*}}^{*}}{\omega_{2^{*}}^{*}}\right|$ & $\left|\frac{\Delta \omega_{3^{*}}^{*}}{\omega_{3^{*}}^{*}}\right|$ & $\left|\frac{\Delta \phi_{12}}{\phi_{12}}\right|$ & $\left|\frac{\Delta \psi_{12}}{\psi_{12}}\right|$ & $\left|\frac{\Delta \alpha}{\alpha}\right|$ & $\left|\frac{\Delta A^{*}}{A^{*}}\right|$ & $\left|\frac{\Delta C^{*}}{C^{*}}\right|$ & $\left|\frac{\Delta k_{2}}{k_{2}}\right|$ \\
\hline $44330-44755$ & 0.10 & $4.6 \mathrm{E}-6$ & $1.3 \mathrm{E}-7$ & $2.3 \mathrm{E}-9$ & 71 & $1.6 \mathrm{E}-3$ & $1.6 \mathrm{E}-2$ & $6.6 \mathrm{E}-4$ & $1.1 \mathrm{E}-3$ & 129 & $1.1 \mathrm{E}-4$ \\
\hline $44970-45403$ & $1.8 \mathrm{E}-6$ & 0.95 & 0.77 & $3.0 \mathrm{E}-5$ & $2.5 \mathrm{E}-7$ & 2.2 & 0.37 & $5.0 \mathrm{E}-2$ & $4.1 \mathrm{E}-6$ & 28 & $1.1 \mathrm{E}-3$ \\
\hline $45700-46135$ & $9.6 \mathrm{E}-6$ & 1.5 & 0.16 & $1.8 \mathrm{E}-6$ & $1.6 \mathrm{E}-6$ & $4.5 \mathrm{E}-8$ & $1.9 \mathrm{E}-10$ & $9.1 \mathrm{E}-6$ & 4.3 & 1.8 & $1.2 \mathrm{E}-5$ \\
\hline $46340-46773$ & $3.0 \mathrm{E}-4$ & 0.44 & 0.11 & $3.7 \mathrm{E}-5$ & 13 & 16 & $2.8 \mathrm{E}-2$ & $3.1 \mathrm{E}-4$ & 4.6 & 7.0 & $6.6 \mathrm{E}-5$ \\
\hline $46977-47410$ & $1.2 \mathrm{E}-6$ & 1.3 & 0.64 & $2.7 \mathrm{E}-5$ & $6.2 \mathrm{E}-7$ & $7.2 \mathrm{E}-8$ & $<1 \mathrm{E}-13$ & $7.9 \mathrm{E}-6$ & 3.8 & 3.5 & $5.8 \mathrm{E}-5$ \\
\hline $47640-48075$ & $3.6 \mathrm{E}-4$ & 3.8 & 0.26 & $2.0 \mathrm{E}-5$ & $1.6 \mathrm{E}-5$ & 6.2 & $3.5 \mathrm{E}-2$ & $7.4 \mathrm{E}-5$ & 4.1 & 1.3 & $1.2 \mathrm{E}-4$ \\
\hline $48260-48696$ & $3.3 \mathrm{E}-5$ & $2.8 \mathrm{E}-2$ & $1.7 \mathrm{E}-3$ & $3.4 \mathrm{E}-5$ & 38 & 26 & $7.7 \mathrm{E}-10$ & $7.0 \mathrm{E}-6$ & 14 & 22 & $4.9 \mathrm{E}-5$ \\
\hline 48900-49336 & $8.3 \mathrm{E}-5$ & 1.1 & 1.9 & $5.0 \mathrm{E}-5$ & 4.6 & 0.34 & 1.4 & $1.5 \mathrm{E}-5$ & 9.9 & 13 & $1.6 \mathrm{E}-4$ \\
\hline 49630-50066 & $<1 \mathrm{E}-13$ & 2.6 & $6.8 \mathrm{E}-2$ & $2.6 \mathrm{E}-7$ & $1.6 \mathrm{E}-3$ & $7.9 \mathrm{E}-14$ & $<1 \mathrm{E}-13$ & $3.2 \mathrm{E}-9$ & 2.7 & 0.38 & $2.5 \mathrm{E}-5$ \\
\hline 50170-50604 & $2.7 \mathrm{E}-5$ & 2.3 & $1.0 \mathrm{E}-6$ & $3.0 \mathrm{E}-6$ & $<1 \mathrm{E}-13$ & $1.1 \mathrm{E}-2$ & $2.2 \mathrm{E}-2$ & $2.7 \mathrm{E}-9$ & 18 & 11 & $1.3 \mathrm{E}-4$ \\
\hline
\end{tabular}

dependent, gravitational field with two contributions, with respect to a $\mathbf{u}_{1} \mathbf{u}_{2} \mathbf{u}_{3}$ reference frame attached to the Earth. The first term is radial:

$a_{r}=\frac{3 G\left(C^{*}-A^{*}\right)}{2 r^{4}}\left[3 \sin ^{2} \theta\left(\cos \phi \sin \omega_{T} t+\sin \phi \cos \omega_{T} t\right)^{2}-1\right]$.
Here, $\theta$ is the co-latitude, $\phi$ is the longitude and $\omega_{T}$ is the Earth angular velocity. Of course, $\omega_{T} t$ is defined except for a phase that would establish the position of the ellipsoid rotation axis with respect to the Greenwich meridian (for instance, in the direction of $\mathbf{u}_{1}$ ), at a given time. The second contribution is

$$
\begin{aligned}
\mathbf{a}_{\theta^{*}}=\frac{3 G\left(C^{*}-A^{*}\right)}{2 r^{4}} \sin 2 \theta^{*}\left[\left(\cos \theta^{*} \sin \phi^{*} \cos \omega_{T} t-\sin \theta^{*} \sin \omega_{T} t\right) \mathbf{u}_{1}\right. \\
\left.-\left(\cos \theta^{*} \sin \phi^{*} \sin \omega_{T} t+\sin \theta^{*} \cos \omega_{T} t\right) \mathbf{u}_{2}+\cos \theta^{*} \cos \phi^{*} \mathbf{u}_{3}\right]
\end{aligned}
$$

where

$$
\begin{aligned}
& \sin \theta^{*}=\sqrt{\cos ^{2} \theta+\sin ^{2} \theta\left(\cos \phi \cos \omega_{T} t-\sin \phi \sin \omega_{T} t\right)^{2}} \\
& \left.\cos \theta^{*}=\sin \theta\left(\cos \phi \sin \omega_{T} t+\sin \phi \cos \omega_{T} t\right)\right)
\end{aligned}
$$

$$
\sin \phi^{*}=\frac{\sin \theta\left(\cos \phi \cos \omega_{T} t-\sin \phi \sin \omega_{T} t\right)}{\sqrt{\cos ^{2} \theta+\sin ^{2} \theta\left(\cos \phi \cos \omega_{T} t-\sin \phi \sin \omega_{T} t\right)^{2}}}
$$

$$
\cos \phi^{*}=\frac{\cos \theta}{\sqrt{\cos ^{2} \theta+\sin ^{2} \theta\left(\cos \phi \cos \omega_{T} t-\sin \phi \sin \omega_{T} t\right)^{2}}} .
$$


TABLE III: Calculated initial values of $\dot{\theta}$ and $\dot{\theta}^{*}$ (in $\mathrm{rad} / \mathrm{s}$ ), with respective appropriate signs, and torque strength $c_{1}$ (in $\mathrm{N} \cdot \mathrm{m}^{2}$ ).

\begin{tabular}{crrc}
\hline interval & \multicolumn{1}{c}{$\dot{\theta}$} & $\dot{\theta}$ & $c_{1}$ \\
\hline & & & \\
$44330-44755$ & $2.426 \mathrm{E}-11$ & $4.141 \mathrm{E}-10$ & $9.226 \mathrm{E}+23$ \\
$44970-45403$ & $-2.684 \mathrm{E}-11$ & $1.419 \mathrm{E}-07$ & $1.367 \mathrm{E}+22$ \\
$45700-46135$ & $-4.176 \mathrm{E}-11$ & $7.080 \mathrm{E}-07$ & $6.790 \mathrm{E}+20$ \\
$46340-46773$ & $3.218 \mathrm{E}-11$ & $3.487 \mathrm{E}-06$ & $5.006 \mathrm{E}+20$ \\
$46977-47410$ & $-2.231 \mathrm{E}-11$ & $6.839 \mathrm{E}-07$ & $6.349 \mathrm{E}+20$ \\
$47640-48075$ & $3.367 \mathrm{E}-11$ & $2.891 \mathrm{E}-06$ & $3.922 \mathrm{E}+20$ \\
$48260-48696$ & $-4.771 \mathrm{E}-12$ & $-4.521 \mathrm{E}-09$ & $2.607 \mathrm{E}+21$ \\
$48900-49336$ & $-9.816 \mathrm{E}-12$ & $1.147 \mathrm{E}-08$ & $2.517 \mathrm{E}+21$ \\
$49630-50066$ & $-5.563 \mathrm{E}-11$ & $3.185 \mathrm{E}-10$ & $3.331 \mathrm{E}+20$ \\
$50170-50604$ & $-4.110 \mathrm{E}-11$ & $4.946 \mathrm{E}-10$ & $1.900 \mathrm{E}+21$
\end{tabular}

TABLE IV: CW periods resulting from the model in comparison with the values from Höpfner [11] (in days).

interval calculated period Höpfner's period

$\begin{array}{lll}44330-44755 & 424.578 & 424.76 \\ 44970-45403 & 432.454 & 432.45 \\ 45700-46135 & 434.677 & 434.68 \\ 46340-46773 & 433.366 & 433.37 \\ 46977-47410 & 433.331 & 433.33 \\ 47640-48075 & 435.123 & 435.12 \\ 48260-48696 & 436.414 & 436.41 \\ 48900-49336 & 436.943 & 436.93 \\ 49630-50066 & 436.924 & 436.92 \\ 50170-50604 & 433.920 & 433.92\end{array}$

Note that, in the Earth's reference frame, the DM ellipsoid gives a complete turn in a day. Supposing the DM ellipsoid is smaller than the Earth core, this gravitational field certainly enforces viscous flow in the outer core, which is in a fluid state. As a first consequence, it can provide the necessary energy to the magneto-hydrodynamic motion in the outer core, which is closely related to the generation of the geomagnetic field. This could give a plausible explanation to one of the basic problems of geophysics (see Stacey, ref. [13]), namely, what is the source of this energy. However the numerical solution of this motion through the Navier-Stokes and energy conservation equations suffers lack of knowledge of the flowing material density dependence with respect to pressure and temperature. Secondly, it can generate heat in the core, that would contribute to decrease the shortfall of $0.7 \mathrm{TW}$ to the energy necessary to maintain the adiabatic temperature gradient in the core, leaving more power to drive the geomagnetic field dynamo [13]. On the other hand, Mack et al. [28] have concluded that DM is unlikely to contribute not only to Earth's internal heat flow but also to hot-Jupiter exoplanets. However they studied the contribution of DM self-annihilation to heat generation, what is a different idea from ours. Conversely, Adler [9] has also studied the contribution of self-annihilating and non-self-annihilating DM accretion to the internal heat of the Earth, Jovian planets and hot-Jupiter exoplanets. His conclusion is that this process is plausible provided efficient DM capture is occurring. In our description, it is feasible to imagine DM ellipsoids also present is such bodies, generating heat by enforcing internal viscous flow, like in Earth. Therefore this model can mean much more than mere emulation of known suggested causes for CW but it also touches open questions like geomagnetic field dynamo and heat generation in the Earth's outer core and in other planets.

Rather than giving the final answer for the problem that has raised the attention of geophysicists and astronomers for several decades, we expect that this calculation may open room for a new and potentially important key component in $\mathrm{CW}$ which was completely unsuspected untill now, to be considered in more sophisticated models. Future development will decide about the real significance of this approach that might have relevant consequences in our comprehension about dark matter. At least, it has been demonstrated that two interacting ellipsoids can have Chandler-like wobble.

\section{ACKNOWLEDGMENTS}

The author would like to thank Prof. Joachim Höpfner (retired from GFZ-Potsdam) for enlightening correspondence and for providing unpublished results, Prof. Kleber C. Mundim, from Chemistry Institute-University of Brasilia, for allowing the use of his GSA code, Prof. Nivaldo A. Lemos, from Universidade Federal Fluminense, for correspondence in the early stages of this work, Prof. Marcos D. Maia, from Institute of Physics-University of Brasilia, for his interest and for pointing me some related literature, Prof. Marcus B. Lacerda Santos, also from Institute of Physics-University of Brasilia, for stimulating discussions, and MEC-SESu for tutorship (PET program). Finally, thanks are due to the Referee of this paper, for interesting remarks.
[1] A. E. H. Love, Proceedings of the Royal Society of London A 82, 73 (1909).

[2] J. Larmor, Proceedings of the Royal Society of London A 82, 89 (1909).

[3] M. L. Smith and F. A. Dahlen, Geophysical Journal of the Royal Astronomical Society 64, 223 (1981).

[4] R. S. Gross, Geophysical Research Letters 27, 2329 (2000).
[5] F. Seitz, J. Stuck and M. Thomas, Geophysical Journal International 157, 25 (2004).

[6] C. D. Froggatt and H. B. Nielsen, Physical Review Letters 95, 231301 (2005).

[7] J.-M. Frère, F.-S. Ling and G. Vertongen, Physical Review D 77, 083005 (2008).

[8] J. Diemand, M. Kuhlen, P. Madau, M. Zemp, B. Moore, D. 
Potter and J. Stadel, Nature 454, 735 (2008); arXiv:astro$\mathrm{ph} / 0805.1244$.

[9] S. L. Adler, arXiv:astro-ph/0808.2823.

[10] S. Khalil and C. Muñoz, Contemporary Physics 43, 51 (2002); arXiv:hep-ph/0110122.

[11] J. Höpfner, Journal of Geodynamics 36, 369 (2003).

[12] J. Höpfner, Surveys in Geophysics 25, 1 (2004).

[13] F. D. Stacey, Physics of the Earth (Brookfield Press, Brisbane, 1992).

[14] A. M. Dziewonski and D. L. Anderson, Physics of the Earth and Planetary Interiors 25, 297 (1981).

[15] K. R. Symon, Mechanics (Addison-Wesley, Reading, 1971).

[16] W. M. Kaula, An Introduction to Planetary Physics (Wiley, New York, 1968).

[17] R. S. Gross, Combinations of Earth orientation measurements: SPACE2004, COMB2004, and POLE2004, JPL-NASA publication 05-6 (Pasadena, California, 2005); ftp://euler.jpl.nasa.gov/keof/combinations/2004.

[18] W. H. Press, B. P. Flannery, S. A. Teukolsky and W. T. Vetterling, Numerical Recipes in Fortran 77 (Cambridge University Press, Cambridge, 1999).
[19] K. C. Mundim and C. Tsallis, International Journal of Quantum Chemistry 58, 373 (1996); preprint http://www.unb.br/iq/kleber/GSA/gsa-java/index.htm.

[20] A. Dall'Igna Junior, R. S. Silva , K. C. Mundim and L. E. Dardenne, Genetics and Molecular Biology 27, 616 (2004).

[21] L. T. Liu, H. T. Hsu, B. X. Gao and B. Wu, Geophysical Research Letters 27, 3001 (2000).

[22] W. J. Wang, Geophysical Journal International 158, 1 (2004).

[23] R. O. Vicente and C. R. Wilson, Journal of Geophysical Research 102, 20439 (1997).

[24] H. Jochmann, Journal of Geodesy 77, 454 (2003).

[25] D. C. Liao and Y. H. Zhou, Chinese Journal of Astronomy and Astrophysics 4, 247 (2004).

[26] J. Y. Guo, H. Greiner-Mai, L. Ballani, H. Jochmann and C. K. Shum, Journal of Geodesy 78, 654 (2005).

[27] F. Gilbert and A. M. Dziewonski, Philosophical Transactions of the Royal Society of London A 278, 187 (1975).

[28] G. D. Mack, J. F. Beacom and G. Bertone, Physical Review D 76, 043523 (2007). 